\title{
MS12-P04 | How Disease-Associated Mutations May Alter the Dynamic Motion of the N-terminal Domain of the Human Cardiac Ryanodine Receptor
}

Bauer, Jacob (Institute of Molecular Biology, SAS, Bratislava, SVK); Pavlovic, Jelena (Institute of Molecular Biology, SAS, Bratislava, SVK); Kutejová, Eva (Institute of Molecular Biology, SAS, Bratislava, SVK); Bauerova, Vladena (Institute of Molecular Biology, SAS, Bratislava, SVK)

The cardiac Ryanodine receptor (RyR2), one of the largest known ion channels, releases $\mathrm{Ca}^{2+}$ ions from the sarcoplasmic reticulum of cardiomyocytes into the cytoplasm, thereby triggering heart cell contraction [1]. Mutations to this channel are associated with inherited cardiac arrhythmias and appear to cluster in distinct parts of the N-terminal, central, and C-terminal areas of the channel [2]. Starting with our previous structure of the human RyR2 N-terminal domain (NTD) [3], we used molecular dynamics simulation to study how the R414L, I419F and R420W mutations affect its structure and dynamics. The R414L and I419F mutations diminish the overall amplitude of motion without greatly changing its direction; R420W both enhances the amplitude and changes the direction of motion. These results suggest that R414L and I419F hinder channel closing, while R420W may enhance channel opening. It appears that the wild-type protein possesses a moderate level of flexibility which allows the gate to close and not open easily without an opening signal. These mutations disrupt this balance by making the gate either too rigid or too loose, making closing difficult or less effective [4].

This work was supported by VEGA research grant 2/0140/16 from the Slovak Grant Agency.

[1] K. Otcu, et al. (1990) J. Biol. Chem. 265, 13472-13483

[2] J. T. Lanner et al. (2010) Cold Spring Harb. Perspect. Biol. 2, a003996

[3] L'. Borko et al. (2014) Acta Cryst. D70, 2897-2912

[4] J. A. Bauer et al. (2019) J. Biomol. Struct. Dyn. (in press) 10.1080/07391102.2019.1600027 